\title{
Input Methods for the Borg-RPE-Scale on Smartwatches
}

\author{
Janko Timmermann, Wilko Heuten \\ OFFIS - Institute for Information Technology \\ Oldenburg, Germany \\ janko.timmermann@offis.de, heuten@offis.de
}

\author{
Susanne Boll \\ University of Oldenburg \\ Oldenburg, Germany \\ susanne.boll@informatik.uni-oldenburg.de
}

\begin{abstract}
The assessment of the perceived exertion during physical activity can be a valuable addition to a healthy training. Modern smartwatches provide a good opportunity to asses the perceived exertion, but entering information is quite challenging due to the very small displays. In this paper, we present an evaluation of five different input methods for entering the perceived exertion on the Borg-RPE scale on smartwatches. Our evaluation shows that speech input is inappropriate for entering the rating while using ranges is promising.
\end{abstract}

Keywords-perceived exertion; borg-rpe-scale; smartwatch; android wear; input

\section{INTRODUCTION}

One main element of cardiac rehabilitation is physical activity, which helps to improve the overall constitution of the patient and can be seen as a core component of the cardiac rehabilitation. In fact, a minimum of 30 minutes of moderate-intensity physical activity reduces the coronary risk factors and increases the patient's well-being [1]. However, the right intensity is hard to determine because of many important influencing factors like age, constitution, weight, health, and medication [2], [3].

Quantitative scales have been developed to overcome the subjective nature of exertion. In cardiac rehabilitation, the Borg-RPE-Scale, a scale ranging from 6 (lowest exertion) to 20 (highest exertion), is used most frequently. Patients can rate their perceived exertion using this scale. This rating can then be used for reflecting the training, adjustments regarding the intensity, or even as main parameter for training adoption [4], so patients can do their training in a comfortable way. In practice, during supervised training, trainers verbally ask patients about their perceived exertion and enter the value on the actual scale. While this is fine for supervised indoor training, patients in unsupervised outdoor studies are asked to record the value on their own without any assistance. However, to assess and record the perceived exertion during an exercise on ones own is quite challenging. To stop and enter the required information is not an option, as this potentially changes the perceived exertion and patients might lose their preferred training pace. Due to the lack of easy and fast to use input techniques, perceived exertion is hardly recorded in outdoor studies, which limits the outdoor studies' success and puts the patients' well-being at risk.

In this paper, we address this problem by evaluating methods of input of ratings on the Borg-RPE-Scale on a smartwatch. Smartwatches are easy and comfortably wearable.

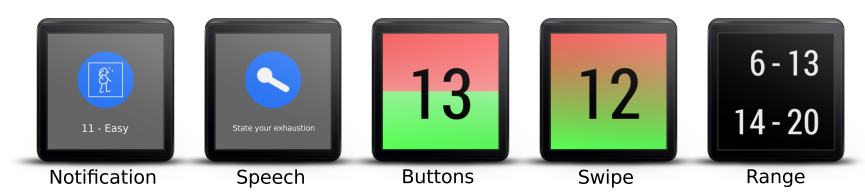

Fig. 1. Input methods created for this study.

User input of information into the smartwatch remains a challenge, since the display has to be small for the device to be easy and comfortable to wear. The well-known input methods for numbers on smart phones, such as drop down lists or keyboards, are hardly usable on smartwatches. We present five input methods with different interaction designs optimized for smartwatches: Using notification buttons, buttons, swipe gestures, speech, and range input. Our study showed that speech input is inappropriate for entering numbers, while using ranges for entering the number is very promising.

\section{RELATED WORK}

Xiao et al. (2014) showed how interaction with smartwatches can be extended by providing mechanical pan, twist, tilt, and click functionality [5]. Among other examples, they showed how the alarm could be set on a smart watch by tilting the display. This scenario is similar to ours. The tilt gesture could be used to change the number the user wants to enter into the smartwatch. Perrault et al. (2013) and Funk et al. (2014) showed how the wristband can be used for interacting with the smartwatch for scrolling or text input [6], [7]. This method could also be interesting in our scenario for future work. In this paper, we focus on available technology and interaction techniques for Android Wear devices. Dunlop et al. (2014) presented an alternate keyboard for a smartwatch display that consisted of six keys for text input [8]. Oney et al. (2014) used a full keyboard on a smartwatch, but used zooming to enlarge the possible letter the user tries to hit after she or he taps the keyboard [9]. In our work, with the Range Input method we used a similar approach adapted to the Borg-RPE-Scale, where the user can choose the desired number using multiple taps.

\section{APPROACH}

According to the Android Wear design principles ${ }^{1}$, an input into a smartwatch should not take more time than five seconds, and the interaction with the watch should be based on big

\footnotetext{
${ }^{1}$ http://developer.android.com/design/wear/principles.html
} 
gestures instead of precise input. Using this guideline, we created five different input methods (Fig. 1) for the Borg-RPEScale on an Android Wear device based on tap and swipe gestures and using the speech input feature. The input was preceded by a notification within the notification stream of the smartwatch. Notification in Android Wear consist of at least one text field with optional graphical background and an arbitrary number of action buttons. Only one text field or action button is presented on the display at a time. The others can be reached by swiping left or right on the watch. For further information about the notification design on Android Wear smartwatches, please refer to the official documentation by Google.

Notification Button Input The Notification Button Input realizes the number input through the requesting notification itself. We added one action button for every value on the Borg-RPE-Scale. Those action buttons are attached to the notification card and can be accessed by swiping left. As icon for the action, we used a visualization for the exertion level. The description contained the number and a textual description of the Borg-RPE-Value. After an action button was pressed, a dialog was shown displaying the chosen value. The Notification Input was realized using native system functions for creating notifications. A request to the user designed like this is automatically consistent with the Android Wear system itself.

Speech Input The Speech Input used the voice input feature of Android Wear devices, which is the standard text input method of Android Wear watches. After pressing the action icon of the notification, the standard voice input dialog of Android Wear appeared and waited for a number spoken by the user.

Button Input The Button Input allowed users to enter a number by tapping buttons to raise or lower an initial number. The buttons were realized as touchable fields in the upper and lower half of the display, to maximize the interaction area and ease the input. The selected number was displayed as big text in front of the buttons. The initial number was located in the center of the Borg-RPE-Scale, i.e. 13. The buttons raised (button on top) or lowered (button on bottom) the value by one with every tab. A longer press did not make a difference. The raise button was colored red, because a higher value on the Borg-RPE-Scale is associated with a higher exertion and the lower button was colored green. After the first interaction with one of the buttons, this method finished the input if no interaction happened for three seconds.

Swipe Input The Swipe Input allowed users to enter a number using swipe gestures which are very common on touch screen devices. It was similar to the Button Input, but used the swiping up gesture for raising the number and the swiping down gesture for lowering the number. Swiping from one end to another on the display changed the value about seven steps. The background was a color gradient from red (top) to green (bottom) to show which direction is associated with raising or lowering the value. All other details were the same as for the Button Input method.

Range Input The Range Input allowed the selection of a value by tapping as the Button Input method, but used the binary search method to minimize the taps. It used ranges that

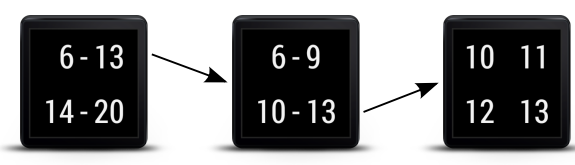

Fig. 2. Example for entering a number using the Range Input method.

the users could tap to refine their input. Like the Button Input, this method had two touchable areas at the top and bottom of the display. Each of the areas showed one part of the scale, e.g. $6-13$ and $14-20$. After the user selected one of these ranges, that range was again split up into two ranges, e.g. 6 -9 and $10-13$. In the last step, the display was split into four touchable areas to choose between the remaining four values. This example is visualized in Fig. 2. To visualize and clarify the change on the screen after the user chose one range, the transition between the current and the new display was animated with a short animation of 500 milliseconds. In our scenario, each value on the Borg-RPE-Scale could be chosen with three taps on the display.

All methods allowed reviewing and correcting the input. Users were able to review their input within a small time window of three seconds and cancel the input, before it was automatically accepted and the notification was removed. If the input was canceled, the users were brought back to the number input to correct their choice.

\section{STUDY}

To evaluate and compare the five different input methods, we conducted a user study. It showed that the range input was the most and the speech input the least popular method.

\section{A. Apparatus}

We used two different smartwatches in our study: The Samsung Gear Live and the LG G Watch. Both have very similar technical specifications and form factors. Users could use their own smartphone during the study. If we provided one, we used a Nexus 5 device.

\section{B. Participants}

We conducted the study with 22 participants (18 male, 4 female). The age ranged from 21 to 50 years. The mean age was 31.73 years (SD: 7.28 years). The participants were recruited within our institute and were not paid for their participation.

\section{Design}

The goal of this study was to compare the five described input methods focused on the aspects: intuitivity, inconvenience, speed, usability during activity, and general applicability. The study started with a pre-questionnaire about demographics and prior knowledge. In particular, we used four statements rated on a five point Likert scale: "I am experienced with smartphones.", "I am experienced with smartwatches.", "I can easily use devices with touchscreen.", and "I know the meaning of the Borg-RPE-Scale.". Additionally, we used two yes/no statements: "I used the Borg-RPE-Scale before." and "I observed the intensity of my physical activity before.". 
Every user used the watch for 24 hours and should wear it whenever possible, except during sleep. We decided to do a field study instead of a lab study since the complexity of problems raised while entering Borg-RPE-Values on a watch seemed to be to hard to simulate satisfyingly. The smartwatch created notifications to enter Borg-RPE-Value at least every 45 minutes. This interval was shortened to a minimum of 10 minutes depending on the accelerometer values measured by the watch, to ask the user in more active situations which is likely in actual use cases of the Borg-RPE-Scale. The notifications were signaled by a stream card on the smartwatch and by three long vibration impulses. If the user did not respond to a notification, again three long vibration impulses were given every three minutes. The method users had to use to report their perceived exertion changed every time. The order was randomized and it was ensured all methods were used equally often.

After 24 hours, the study ended with a post-questionnaire where the participants rated five statements on a five-point Likert scale for every input method: "The input method was intuitive.", "The input method was laborious.", "The input was fast.", "The input method can be used during physical activity.", and "Overall I think this input method was suitable for the input.". The questionnaire depicted all input methods to ease remembering them. It also contained white space for every input method, so users were able to write down other comments. Finally, the participants ranked the input methods from best to worst.

\section{Procedure}

In the beginning of the study, we met the participants at a previously communicated location, gave the study description to them, and let them sign the informed consent. The participants filled out the first questionnaire about demographics and prior knowledge. Afterward, we explained the smartwatch, i.e. how it is generally used. After they understood the general usage of the smartwatch, we demonstrated each of the five input methods and they were able to try them out as often as they liked. We also showed the second questionnaire to them, so they knew beforehand how the input methods had to be rated in the end. If everything was clear, the study was started and the participants took the smartwatch and the smartphone with them. After 24 hours, we met again and the participants handed back the devices to us. After they filled out the second questionnaire, we asked for further comments about the study. In the end, we thanked the participants for their effort and ended the study.

\section{E. Results}

For the evaluation of the replies on the Liekert scale, we used 0 for strongly agree and 4 for strongly disagree. The participants rated the statement "I am experienced with smart phones." with 0 (Min: 0, Max: 2, 1st Qu.: 0, 3rd Qu.: 0) as median rating. The statement "I can easily use devices with touch screen." was consequently rated very similarly with 0 (Min: 0, Max: 3, 1st Qu.: 0, 3rd Qu.: 0) as median rating. The experience with smartwatches was much lower and was rated with 3 (Min: 0, Max: 4, 1st Qu.: 3, 3rd Qu.: 3) as median rating. The statement "I know the meaning of the Borg-RPE-Scale." was rated with 1 (Min: 0, Max: 4, 1st Qu.: 1,
TABLE I. MEDIAN RATINGS FOR INTUITIVITY, INCONVENIENCE, SPEED, USABILITY DURING ACTIVITY, AND GENERAL APPLICABILITY

\begin{tabular}{l||c|c|c|c|c} 
& intuitive & inconv. & speed & activity & general \\
\hline Notifi. & 0 & 3 & 1 & 2 & 1 \\
\hline Speech & 0 & 2 & 1 & 1 & 2 \\
\hline Buttons & 0 & 3 & 1 & 1 & 1 \\
\hline Swipe & 1 & 3 & 1 & 1 & 1 \\
\hline Range & 0 & 4 & 0 & 0.5 & 0 \\
\hline
\end{tabular}

3rd Qu.: 2.75) as median rating. However, only one participant already used the Borg-RPE-Scale before.

Overall, the exertion was entered 818 times during our study, resulting in an average of 37.2 (SD: 15.2) inputs per participant. Thus, every input method was on average used 7 to 8 times per user. The participants rated all input methods regarding intuitivity, inconvenience, subjective speed, usability during activity, and general applicability using the statements described in the Design section. The median ratings are depicted in TABLE I. We used the Friedman test to check for significant differences of the rating between our input methods. The test indicated no differences for inconvenience $(p=0.24)$, and subjective speed $(p=0.1)$, but for intuitivity $(p<0.001)$, usability during activity $(p<0.05)$, and the general applicability $(p<0.05)$. We used the Wilcoxon rank sum test with continuity correction for pairwise comparisons of the input methods for these three aspects and corrected the resulting $\mathrm{p}$-values using the Bonferroni correction. We found no significant differences for usability during activity and the general applicability with the pairwise comparisons. The intuitivity differed significantly between the swipe and speech input methods $(p<0.05, z=-0.34)$.

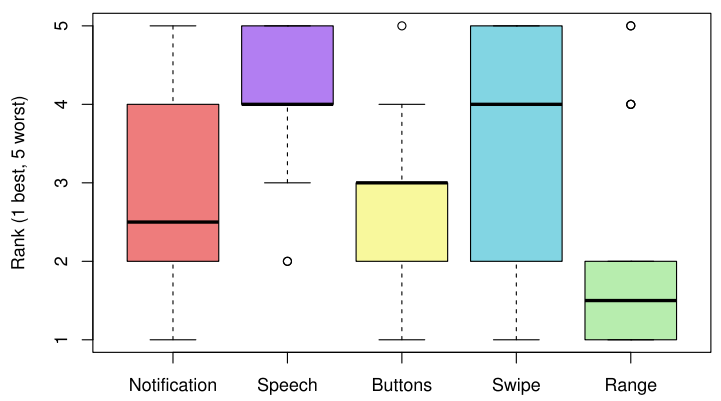

Fig. 3. Ranking of the input methods (1 best, 5 worst).

The participants also ranked the input methods from 1 to 5 , without ties being allowed. The median, minimum, maximum, and quantiles of the rankings are depicted in Fig. 3. The range input method was rated on the first rank by eleven participants, the swipe method by five, the notification button method by four, and the button method by two. The speech input was never rated best. The Friedman test indicated significant differences between the input methods $(p<0.001)$. We used the Wilcoxon ranked sum test with continuity correction for pairwise comparisons between the input methods and corrected the resulting p-values using the Bonferroni correction. The pairwise comparisons showed a significant difference between the ranking of the button input and the speech input $(p<0.05$, $z=-0.26)$, as well as between the range input and the speech input $(p<0.01, z=-0.44)$. The speech input was especially vulnerable for input errors due to possible errors in speech recognition. During our study, we recorded an average 


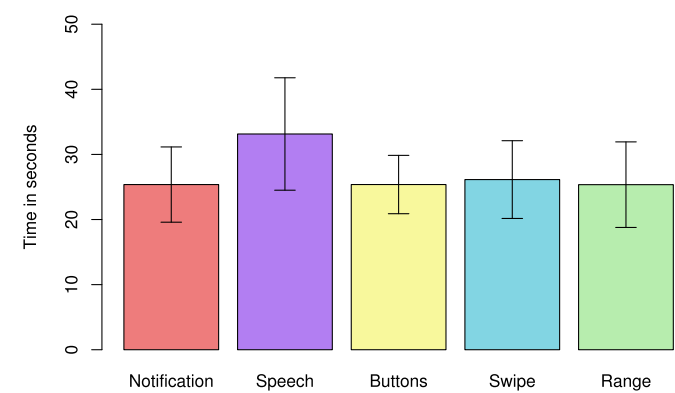

Fig. 4. Time needed by the participants for entering a value using the different methods. Error bars show the $95 \%$ confidence interval.

\section{of $13.9 \%$ of false recognized speech per user.}

We measured the time it took the participants to enter their perceived exertion. The notification button method as well as the speech input method were realized using system functions, so we were not able to measure the time of the user input alone. Because of this limitation, the time for all methods was measured from the time the notification was given to the user until the value was entered. This way we were able to obtain comparable measurements for all methods, but also included possible time it took the participant to react to the notification. However, this affected all input methods in the same way. Fig. 4 shows the average time needed for entering a value using the different methods. We used the Quade test on these data which indicated no significant differences between the five input methods $(p=0.59)$.

Additional qualitative feedback was given mainly for the speech input, where eight participants explicitly stated that they were annoyed by false recognition and nine reported that they felt awkward when they had to use it in public. However, we also got reports about the speech recognition working very well. The swipe input was criticized for the ambiguity about which direction leads to higher and lower values, but alongside the button method it was praised for starting in the middle of the scale, which eased the input.

\section{Discussion}

All input methods had decent ratings among all our categories. Regarding intuitivity, we saw that the median rating for the swipe input was 1 (agree) and for all other input methods 0 (completely agree). Despite the graphical hint in the background, the swipe input showed the weakness that some participants were confused in what direction they had to swipe to change the value the way they wanted. Most participants stated that changing the direction would not help them, but over time they will get used to it.

The speech input was the most inconvenient input method. For our participants, it was obviously annoying when the speech recognition did not work well and the input had to be repeated. Especially if the situation is inappropriate, repeating the speech input gets more and more annoying. The social acceptance for speech input may change in the future due to the upcoming smartwatch technology and ease the use of speech input in public. However, public environments tend to be more noisy which makes the speech recognition more complicated and less accurate.
The ratings for the other categories were quite mixed, but we saw that the range method got very good ratings in all categories. This is surprising, because many participants were skeptical about that input method in the beginning of the study. However, the constant effort for entering a value, i.e. always exactly three taps, seemed to be a good user experience in our scenario.

Our results showed no significant difference for objective input speed, which is consistent to the subjective rating of the users regarding the input speed which also showed no significant differences between the input methods. While we had to include the response time to the notification by the user for the objective measurement, the variance may be raised and small changes among the input techniques became undetectable. However, we can assume that no relevant large differences exist between the input methods.

\section{CONCLUSiON}

In this paper, we presented five different input methods for entering the perceived exertion using the Borg-RPE-Scale on Android Wear smartwatches. In a user study we tested these methods and found that speech input is the worst and range input is the best method in our chosen scenario. The best input method may strongly depend on the context, but our results show clear advantages of using ranges for entering the exertion. While our input methods are specialized for entering values on the Borg-RPE-Scale, our results still provide useful basic findings for the new area of smartwatch interaction.

\section{REFERENCES}

[1] G. Balady, M. Williams, P. Ades, V. Bittner, P. Comoss, J. Foody, B. Franklin, B. Sanderson, and D. Southard, "Core components of cardiac rehabilitation/secondary prevention programs: 2007 update," Circulation, vol. 115 , no. 20, pp. 2675-2682, 2007.

[2] G. Borg, Borg's perceived exertion and pain scales. Human Kinetics Publishers, 1998.

[3] — , "Anstrengungsempfinden und körperliche aktivität," Deutsches Ärzteblatt, vol. 101, no. 15, pp. 1016-21, 2004.

[4] J. Timmermann, A. Workowski, W. Heuten, D. Willemsen, and S. Boll, "Human centered training: perceived exertion as main parameter for training adaption," in Proceedings of the 8th Nordic Conference on Human-Computer Interaction. ACM, 2014, pp. 995-998.

[5] R. Xiao, G. Laput, and C. Harrison, "Expanding the input expressivity of smartwatches with mechanical pan, twist, tilt and click," in CHI'14 Proc. ACM, 2014, pp. 193-196.

[6] S. T. Perrault, E. Lecolinet, J. Eagan, and Y. Guiard, "Watchit: simple gestures and eyes-free interaction for wristwatches and bracelets," in CHI'13 Proc. ACM, 2013, pp. 1451-1460.

[7] M. Funk, A. Sahami, N. Henze, and A. Schmidt, "Using a touch-sensitive wristband for text entry on smart watches," in CHI'14 Extended Abstracts on Human Factors in Computing Systems. ACM, 2014, pp. 2305-2310.

[8] M. D. Dunlop, A. Komninos, and N. Durga, "Towards high quality text entry on smartwatches," in CHI'14 Extended Abstracts on Human Factors in Computing Systems. ACM, 2014, pp. 2365-2370.

[9] S. Oney, C. Harrison, A. Ogan, and J. Wiese, "Zoomboard: a diminutive qwerty soft keyboard using iterative zooming for ultra-small devices," in CHI'13 Proc. ACM, 2013. 